\title{
ON THE TRANSITION FROM ACCRETION-POWERED TO ROTATION-POWERED MILLISECOND PULSARS
}

\author{
J. TAKATA ${ }^{1}$, K. S. Cheng ${ }^{1}$, AND Ronald E. TAAM ${ }^{2,3}$ \\ ${ }^{1}$ Department of Physics, University of Hong Kong, Pokfulam Road, Hong Kong; takata@hku.hk; hrspksc@hkucc.hku.hk \\ ${ }^{2}$ Department of Physics and Astronomy, Northwestern University, 2131 Tech Drive, Evanston, IL 60208, USA; r-taam@northwestern.edu \\ ${ }^{3}$ Academia Sinica Institute of Astronomy and Astrophysics-TIARA, P.O. Box 23-141, Taipei 10617, Taiwan \\ Received 2010 August 16; accepted 2010 September 27; published 2010 October 12
}

\begin{abstract}
The heating associated with the deposition of $\gamma$-rays in an accretion disk is proposed as a mechanism to facilitate the transformation of a low-mass X-ray binary to the radio millisecond pulsar (MSP) phase. The $\gamma$-ray emission produced in the outer gap accelerator in the pulsar magnetosphere likely irradiates the surrounding disk, resulting in its heating and the possible escape of matter from the system. We apply the model to PSR J1023+0038, which has recently been discovered as a newly born rotation-powered MSP. The predicted $\gamma$-ray luminosity $\sim 6 \times 10^{34} \mathrm{erg} \mathrm{s}^{-1}$ can be sufficient to explain the disappearance of the truncated disk existing during the 8 month $-2 \mathrm{yr}$ period prior to the 2002 observations of $\mathrm{J} 1023+0038$ and the energy input required for the anomalously bright optical emission of its companion star.
\end{abstract}

Key words: binaries: close - magnetic fields - pulsars: individual (PSR J1023+0038) - stars: neutron

\section{INTRODUCTION}

The discovery of pulsed radio emission at $1.69 \mathrm{~ms}$ from the source J102347.67+003841.2 (see Archibald et al. 2009), offers a unique opportunity to study the evolution of a system from the low-mass X-ray binary (LMXB) phase to the recycled millisecond pulsar (MSP) phase. The system has an orbital period of $4.75 \mathrm{hr}$ (see Woudt et al. 2004) and is distinguished from other systems by a change in optical appearance from a spectrum characterized by broad double peaked hydrogen and helium emission lines (see Bond et al. 2002) to a latetype absorption spectrum (see Thorstensen \& Armstrong 2005). Further analysis based on Sloan Digital Sky Survey (SDSS) spectra by Wang et al. (2009) provide evidence for the presence of a truncated accretion disk in the early observations in 2001 and its possible absence in 2002. The recent detection of Xray pulsations by Archibald et al. (2010) suggests that pulsar high-energy emission activity was already present in 2008.

The description of the transition from the accretion-powered to the rotation-powered phase is not understood due to the complexities in the description of the interaction between the magnetosphere of a neutron star (NS) and its accretion disk. Simple arguments have been discussed in early work by Campana et al. (1998). Although theoretical developments have not progressed significantly, recent observations of the optical counterparts of NS soft X-ray transients in the quiescent state have provided indirect evidence for additional heating of the companion star. As examples, orbital modulations in the optical emission from J102347.68+003841.2 (Thorstensen \& Armstrong 2005), SAX J1808.4-3658 (Burderi et al. 2003; Deloye et al. 2008), XTE 1814-338 (D'Avanzo et al. 2009), and IGR J00291+5934 (Jonker et al. 2008) have been found in their respective quiescent states. Specifically, the amplitude of the modulation cannot be explained by irradiation associated with the $\mathrm{X}$-ray emission from the disk or NS due to insufficient luminosity, indicating the need for the operation of an additional heating source. To provide an explanation of the orbital modulation of the optical emission, pulsar wind models have been suggested in which heating of the donor is due to the effect of a relativistic pulsar wind (Burderi et al. 2003). In addition, the interaction between the pulsar and its companion has been suggested as leading to the formation of an intrabinary shock (see Campana et al. 1998) for the generation of non-thermal X-ray radiation observed in J0024-7204W in the globular cluster 47 Tuc (see Bogdanov et al. 2005), in PSR J1740-5340 in NGC 6397 (Grindlay et al. 2002), and in PSR J1023+0038 (Archibald et al. 2010). Finally, the disappearance of the truncated disk in J102347.67+003841.2 may have been a direct result of the activation of the pulsar.

In order to facilitate this transition, the accretion of matter onto the NS must decrease rapidly, for otherwise, the NS will spin down (e.g., see, Jeffrey 1986). In contrast to the case of radio MSPs in long orbital period systems where a red giant companion can detach from its Roche lobe as its envelope is exhausted, a sudden decrease in the mass transfer from the donor is not generally expected for the donors in short-period binary systems. For these systems, the disk must be truncated sufficiently such that the mass flow is prevented from entering into the NS magnetosphere. A mechanism possibly leading to a sudden decrease in the accretion flow is the operation of the socalled "propeller" effect (Campana et al. 1998; Romanova et al. 2009), in which the Alfvén radius exceeds the co-rotation radius, during the quiescent state of an X-ray transient LMXB phase. In this regime, however, the prevention of matter accreting onto the NS is uncertain. That is, matter can either be swept away or accrete intermittently onto the NS. Ablation due to the action of a pulsar wind may also facilitate the removal of the disk around the pulsar (Wang et al. 2009), however, an important theoretical uncertainty is the description by which a particle kinetic-dominated flow can be formed near the pulsar (Kirk \& Skjæraasen 2003; Arons 2008).

In this Letter, we explore the possibility that irradiation by $\gamma$-ray emission produced in the outer gap accelerator within a pulsar magnetosphere can facilitate dissolution of the disk. In Section 2, we briefly describe the theoretical concepts of the model and apply the theory to J102347.67+003841.2 in Section 3. In the last section, we discuss the implications of our model and conclude.

\section{THEORETICAL MODEL}

The activation of the radio pulsar phase of an NS in a shortperiod LMXB is likely to occur during the quiescent state (see 


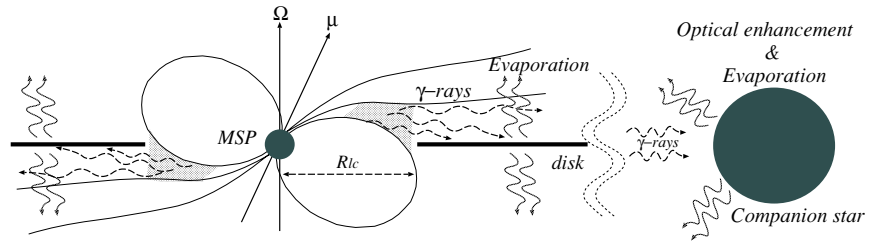

Figure 1. Schematic view of irradiation by the $\gamma$-rays from the outer gap (shadowed region) within a pulsar magnetosphere. The outer gap accelerator is extending between the null charge surface, where $\vec{\Omega} \cdot \vec{B}=0$, to the light cylinder.

Campana et al. 1998) when the mass accretion rates are lower than the time-averaged value, typically $\dot{M} \sim 10^{14}-10^{16} \mathrm{~g} \mathrm{~s}^{-1}$ (Heinke et al. 2009). In such a phase, the magnetosphere can extend beyond the light cylinder radius. If the pulsar magnetosphere is sufficiently clear of matter, $\gamma$-ray photons with an energy $\sim \mathrm{GeV}$ can be produced in an outer gap accelerator (Cheng et al. 1986a, 1986b) via the curvature radiation process of high-energy particles. The emitted $\gamma$-rays can irradiate the accretion disk to promote the escape of disk material from the system (cf. Figure 1). Sufficient heating of the disk may directly lead to the escape of matter or to an increase in its vertical scale height. In this case, the disk would subtend a larger solid angle as seen from the NS, thereby facilitating the acceleration of disk material associated with the radiation pressure of the magnetic dipole radiation. In the following, we describe the process.

\section{1. $\gamma$-ray Emissions from the Outer Gap}

In the outer gap model for the rotation-powered pulsars, particles can be accelerated by the electric field along the magnetic field lines in the region where the local charge density deviates from Goldreich-Julian charge density. The typical strength of the accelerating field in the gap is expressed as (Zhang \& Cheng 1997)

$$
\begin{aligned}
E_{\|} \sim & \frac{f^{2} V_{a}}{s} \sim 9.2 \times 10^{5} f^{2}\left(\frac{P}{10^{-3} \mathrm{~s}}\right)^{-3}\left(\frac{B_{s}}{10^{8} \mathrm{G}}\right)\left(\frac{s}{R_{\mathrm{lc}}}\right)^{-1} \\
& \times\left(\frac{R_{s}}{10^{6} \mathrm{~cm}}\right)^{3}(\mathrm{cgs}),
\end{aligned}
$$

where $V_{a}=B_{s} R_{s}^{3} / R_{\mathrm{lc}}^{2}$ is the electrical potential drop on the polar cap, $P$ is the rotation period, $B_{s}$ is the stellar magnetic field, $s$ is the curvature radius of the magnetic field line, $R_{\mathrm{lc}}=c P / 2 \pi$ is the light cylinder radius, and $R_{s}$ is the stellar radius. In addition, $f$ is the fractional gap thickness, which is defined by the ratio of the gap thickness to $R_{\mathrm{lc}}$. By assuming force balance between the acceleration and radiation reaction of the curvature radiation process, the typical Lorentz factor of the particles in the gap is

$$
\Gamma=\left(\frac{3 s^{2}}{2 e} E_{\|}\right)^{1 / 4} \sim 1.6 \times 10^{7} f^{1 / 2} P_{-3}^{-1 / 4} B_{8}^{1 / 4} s_{1}^{1 / 4} R_{6}^{3 / 4},
$$

where $P_{-3}=(P / 1 \mathrm{~ms}), B_{8}=\left(B_{s} / 10^{8} \mathrm{G}\right), s_{1}=\left(s / R_{\mathrm{lc}}\right)$, and $R_{6}=\left(R_{s} / 10^{6} \mathrm{~cm}\right)$. The corresponding photon energy of the curvature radiation is

$$
E_{\gamma}=\frac{3 h c \Gamma^{3}}{4 \pi s} \sim 25 f^{3 / 2} P_{-3}^{-7 / 4} B_{8}^{3 / 4} s_{1}^{-1 / 4} R_{6}^{9 / 4} \mathrm{GeV} .
$$

The $\gamma$-rays emitted in the gap will be converted into electron/ positron pairs via the photon-photon pair-creation process with
X-ray photons from either the NS surface or the accretion disk. Because the created pairs tend to screen the electric field in the gap, the gap thickness is controlled by the photon-photon pair-creation process. Using the pair-creation condition that $E_{\gamma} E_{X} \sim\left(m_{e} c^{2}\right)^{2}, f$ is estimated as

$$
f \sim 0.22 P_{-3}^{7 / 6} B_{8}^{-1 / 2} s_{1}^{1 / 6} R_{6}^{-3 / 2} E_{0.1}^{-2 / 3},
$$

where $E_{0.1}$ is the typical X-ray photon energy in units of $100 \mathrm{eV}$. The luminosity of the $\gamma$-ray emission from the outer gap is estimated as

$$
L_{\gamma} \sim f^{3} L_{\mathrm{sd}} \sim 4.2 \times 10^{33} P_{-3}^{-1 / 2} B_{8}^{1 / 2} s_{1}^{1 / 2} R_{6}^{3 / 2} E_{0.1}^{-2} \mathrm{erg} \mathrm{s}^{-1},
$$

where $L_{\mathrm{sd}}=4(2 \pi)^{4} B_{s}^{2} R^{6} / 6 c^{3} P^{4}$ is the pulsar spin down power.

The rotation period of the newly switched on MSP is related to the history of the accretion onto the NS. In particular, its rotation period in the accretion stage may be related to the equilibrium spin period, which is obtained by equating the corotation radius, $r_{c o}=\left(G M P^{2} / 4 \pi^{2}\right)^{1 / 3}$ to the magnetospheric radius, $r_{M} \sim 2.4 \times 10^{6} B_{8}^{4 / 7} R_{6}^{12 / 7} \dot{M}_{15}^{-2 / 7} M_{1.4}^{-1 / 7} \mathrm{~cm}$. Here, $M$ is the NS mass, $\dot{M}_{15}$ is the time-averaged accretion rate in units of $10^{15} \mathrm{~g} \mathrm{~s}^{-1}$, and $M_{1.4}$ is the NS mass in units $1.4 M_{\odot}$. However, the spin period may not be equal to its equilibrium spin period since the pressure buildup associated with the accumulation of disk matter at the magnetospheric boundary can push the magnetospheric boundary inside the magnetospheric radius $r_{M}$ (Romanova et al. 2009), resulting in a higher spin period than its equilibrium value. Because of this uncertainty, we introduce a parameter, $\beta$, as $r_{c o}=\beta r_{M}$, which yields the rotation period,

$$
P_{e,-3}=1.7 \kappa B_{8}^{6 / 7} .
$$

where $\kappa=\beta^{3 / 2} R_{6}^{18 / 7} \dot{M}_{15}^{-3 / 7} M_{1.4}^{-5 / 7}$. The spin period is illustrated in Figure 2 as a function of the stellar magnetic field with $\kappa=1$ (solid line), 0.4 (dashed line), and 0.2 (dash-dotted line). For example, with $\beta=0.5$ and $R_{6}=M_{1.4}=1, \kappa=1,0.4$, and 0.2 corresponding to $\dot{M}_{15} \sim 0.1, \sim 0.7$ and $\sim 4$, respectively. In addition, we also plot the observed spin periods and inferred magnetic fields of the radio MSP population in the Galactic field. Only MSPs in binaries with orbital periods less than about 10 days are included since those systems with longer periods may have had red giant companions. In this case, the dissolution of the disk is due to the cessation of mass transfer as the envelope of the giant star is exhausted leading to the shrinkage of the red giant within its Roche lobe.

\section{2. $\gamma$-ray Irradiation of the Disk}

The magnetospheric $\gamma$-rays irradiating the surrounding disk may be absorbed via the so-called pair-creation process in a Coulomb field by nuclei in the accretion disk. Further absorption will occur as the relativistic pairs created with a Lorentz factor $\Gamma \sim 10^{3}$ will transfer their energy and momentum to the disk matter via the Coulomb scattering process.

The cross section of the above pair-creation process is given by Lang (1999) as

$$
\begin{aligned}
\sigma\left(E_{\gamma}\right)= & 3.5 \times 10^{-3} Z^{2} \sigma_{T}\left[\frac{7}{9} \log \left(\frac{183}{Z^{1 / 2}}\right)-\frac{1}{53}\right] \text { for } \\
& \times \frac{E_{\gamma}}{2} \gg \frac{m_{e} c^{2}}{\alpha Z^{1 / 3}}
\end{aligned}
$$




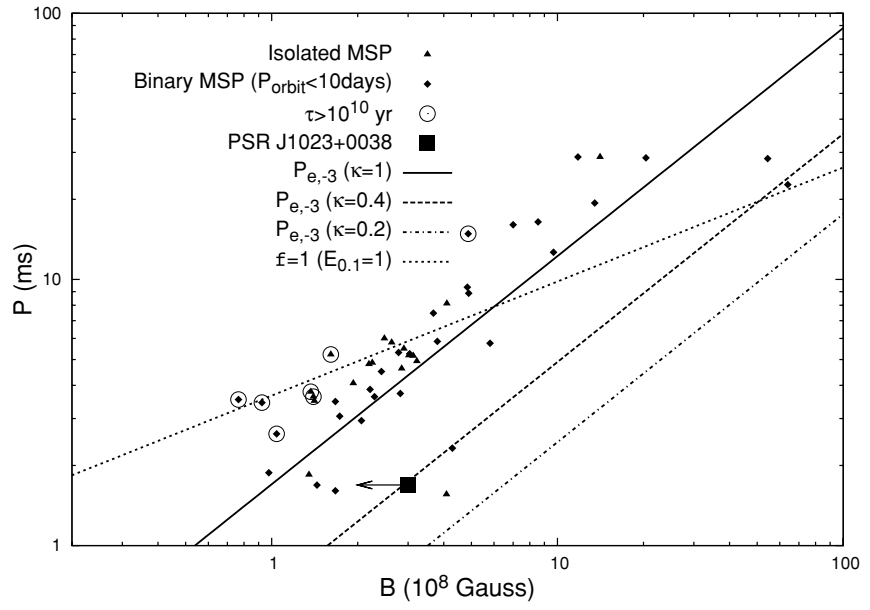

Figure 2. Plot of $P$ vs. $B_{s}$ for the MSPs. The triangles and diamonds represent the field-isolated MSPs and the field binary MSP with an orbital period smaller than 10 days. In addition, the circles show the MSPs with a characteristic age larger than 10 Gyr. The filled square represents the newly born MSP J1023+0038 with $B_{s}=3 \times 10^{8} \mathrm{G}$, which is indicated as an upper limit. The solid, dashed, and dot-dashed line present the relation $P_{e}=1.7 \kappa B^{7 / 6}$ with $\kappa=1, \kappa=0.4$, and $\kappa=0.2$. The dotted line represents the line of $f=1$ with $E_{0.1}=1$. The results are for $R_{6}=M_{1.4}=s_{1}=1$.

where $\sigma_{T}$ is the Thomson cross section, $Z$ is the atomic number, $\alpha$ is the fine structure constant, and $m_{e} c^{2}$ is electron rest mass energy. All $\gamma$-rays irradiating the disk can be absorbed if the column density of the accretion disk exceeds a critical value given by

$$
\Sigma_{\text {crit }} \sim \frac{m_{p}}{\sigma} \frac{h}{l} \sim 60 \frac{h}{l} \mathrm{~g} \mathrm{~cm}^{-2},
$$

where $l$ is the propagation length of the $\gamma$-rays in the disk, $h$ is the thickness of the disk, $Z^{2}=3$ appropriate for a solar abundance, and $m_{p}$ is the mass of a proton. It can be seen that the above column density is smaller than the typical column density of the accretion disk. For the standard gas pressure supported disk model (Frank et al. 2002), the column density is

$$
\Sigma\left(R_{\mathrm{lc}}\right) \sim 2.1 \times 10^{3} \alpha_{0.1}^{-4 / 5} \dot{M}_{15}^{7 / 10} M_{1.4}^{1 / 4} P_{-3}^{-3 / 4} \mathrm{~g} \mathrm{~cm}^{-2},
$$

where $\alpha_{0.1}$ is the viscosity parameter in units of 0.1 . We find that the required column density (8) is much smaller than that of the standard disk model for viscosity parameters less than unity.

An estimate of a critical accretion rate below which the radiation associated with $\gamma$-rays from the outer gap can energetically eject the matter can be obtained by equating $L_{\gamma}=G M M / 2 R_{l c}$ with Equation (5), given by

$$
\dot{M}_{c}=2.1 \times 10^{14} P_{-3}^{1 / 2} B_{8}^{1 / 2} s_{1}^{1 / 2} R_{6}^{3 / 2} E_{0.1}^{-2} M_{1.4}^{-1} \mathrm{~g} \mathrm{~s}^{-1} .
$$

Applying the critical accretion rate (10) and $\beta=0.5$ to Equation (6) and using Equation (5), the predicted $\gamma$-ray luminosity and the surface magnetic field are illustrated as a function of equilibrium period and the X-ray photon energy in Figure 3. The different lines represent the results for the different X-ray photon energies. For a pulsar with $P_{-3}=5$ and $E_{0.1}=0.5$, for example, the predicted $\gamma$-ray luminosity and the surface magnetic field are $L_{\gamma} \sim 5 \times 10^{34} \mathrm{erg} \mathrm{s}^{-1}$ and $B_{8}=41$, respectively. Note that if the true accretion rate is less than the critical accretion rate, the predicted magnetic field and $\gamma$-ray luminosity for the specific set of the rotation period and the $\mathrm{X}$ ray photon energy provide smaller value than those associated with the critical rate.

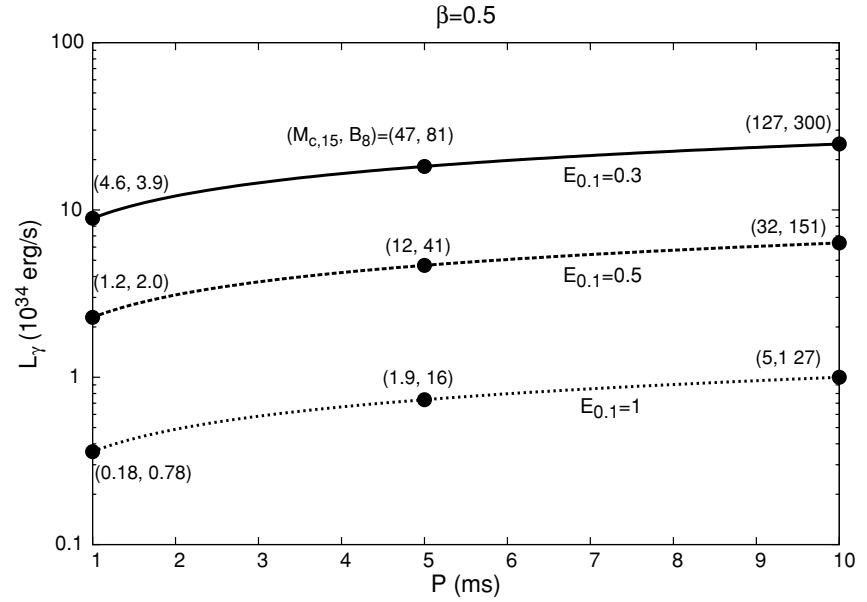

Figure 3. Predicted $\gamma$-ray luminosity and the surface magnetic field at the transition from the accretion-powered to rotation-powered phases. The results are for the accretion rate described by the critical value, that is, $\dot{M}=\dot{M}_{c}$ and $\beta=0.5$. The different lines represent results for the different typical energy of the $\mathrm{X}$-ray photons.

\section{APPLICATION TO PSR J1023+0038}

As an application of our model, we estimate the $\gamma$-ray luminosity which inhibits accretion into the magnetosphere, once the rotation-powered pulsar is activated. In Figure 2, the observed characteristics (filled box) for PSR J1023+0038 are indicated. For the X-ray emission, Homer et al. (2006) found that the X-ray emission in 2004 observations are well fit by an NS atmosphere model $\left(E_{X} \sim 30 \mathrm{eV}\right)$ plus power-law component. However, we choose the X-ray emission from the NS stellar surface rather than the X-ray emission from the pair creation originating from either the pulsar magnetosphere or a shock where material overflowing the companion interacts with the pulsar wind. If the X-ray emission is magnetospheric in origin, the synchrotron emission from the secondary pairs produced outside the main acceleration region is possible. In such a case, because the high-energy photons are propagating to the convex side of the magnetic field lines, the non-thermal X-ray photons are unlikely to illuminate the gap region and, therefore, collisions with the $\gamma$-rays inside the gap are not expected. On the other hand, if the non-thermal X-rays originate from the shock region, the photon-number density in the pulsar magnetosphere is much smaller than that associated with the thermal X-rays from the NS surface, indicating the photon-photon pair-creation process in the gap mainly occurs with the thermal X-rays.

As Figure 2 reveals, the parameter $\kappa \leqslant 0.4$ in the relation (6) can be consistent with the observed rotation period and upper limit of the inferred surface magnetic field. This may indicate that the averaged accretion rate is of the order of $\dot{M} \sim 10^{15} \mathrm{~g} \mathrm{~s}^{-1}$ unless $\beta$ is much smaller than unity. On the other hand, the predicted $\gamma$-ray luminosity (5) and the critical accretion rate (10) give $L_{\gamma} \sim 6 \times 10^{34} \mathrm{erg} \mathrm{s}^{-1}$ and $\dot{M} \sim 5 \times 10^{15} \mathrm{~g} \mathrm{~s}^{-1}$, respectively, with $B_{8}=3$ and $E_{0.1}=0.3$ and $s_{1}=R_{6}=$ $M_{1.4}=1$, indicating that the $\gamma$-ray irradiation on the disk would be sufficient to inhibit the accretion flow into the magnetosphere. We note that the predicted luminosity $L_{\gamma} \sim 6 \times 10^{34} \mathrm{erg} \mathrm{s}^{-1}$ is consistent with the required luminosity from an irradiating source to explain the heating of the companion star (Thorstensen \& Armstrong 2005).

As argued by Wang et al. (2009), there is evidence that a truncated disk existed in SDSS J102347.6+00381 during the 8 month-2 yr period prior to the 2002 observations. Based 
on a standard disk model, a disk extending from $10^{9} \mathrm{~cm}$ to $5.7 \times 10^{10} \mathrm{~cm}$ with a total disk mass of $M_{d} \sim 1.7 \times 10^{23} \mathrm{~g}$ was inferred. Here, we examine whether the purported $\gamma$-ray irradiation can remove the disk within a timescale of less than 2 yr.

Wang et al. (2009) estimate a disk column density of $\Sigma_{d} \sim$ $36(\alpha / 0.1)^{-4 / 5} \dot{M}_{16}^{7 / 10} r_{10}^{-3 / 4}$, which is higher than the required density (Equation (8)) for $\gamma$-ray absorption because the propagation distance in the disk is much longer than the disk thickness. Here, we examine the fraction of the $\gamma$-ray power which can irradiate the disk at $r=10^{9}-10^{10} \mathrm{~cm}$. If we apply (1) a pure dipole magnetic field, (2) the geometry of the outer gap accelerator, which extends from the so-called Goldreich-Julian null charge surface, where the product $\vec{\Omega} \cdot \vec{B}=0$, to the light cylinder, and (3) a standard disk model, implying a disk thickness of $h \sim 10^{8}$ $\mathrm{cm}$ at $r \sim 10^{10} \mathrm{~cm}$ from the pulsar, we find that about $\eta \sim 0.1 \%$ of the outer gap region can illuminate the disk extending beyond $r \geqslant 10^{9} \mathrm{~cm}$. Assuming that the $\gamma$-ray irradiation can remove the matter at a rate of $\dot{M}=\eta L_{\gamma} r / G M$, the timescale of the life of the disk, $\tau \sim M_{d} / \dot{M}$, is

$$
\tau \sim 1\left(\frac{\eta L_{\gamma}}{6 \cdot 10^{31} \mathrm{erg} \mathrm{s}^{-1}}\right)^{-1}\left(\frac{r}{10^{10} \mathrm{~cm}}\right)^{-1}\left(\frac{M_{d}}{10^{23} \mathrm{~g}}\right) M_{1.4} \mathrm{yr}
$$

which can be consistent with the life time inferred from the observation.

\section{DISCUSSION AND CONCLUSION}

Since our scenario for describing the phase from the accreting MSP phase to the radio MSP phase invokes the $\gamma$-ray radiation from the outer gap, observations using the Fermi satellite provide important constraints on the $\gamma$-ray emission model. Since the spin down power of PSR J1023+0038 $\left(L_{\mathrm{sd}} \leqslant 3 \times 10^{35} \mathrm{erg} \mathrm{s}^{-1}\right)$ is relatively high compared to $\gamma$-ray emitting MSPs detected by Fermi (Abdo et al. 2009), and its distance ( $d \sim 1 \mathrm{kpc}$ ) is similar to these Fermi sources, PSR J1023+0038 is a prime candidate for $\gamma$-ray emission.

Recently, Tam et al. (2010) report the discovery of the $\gamma$ ray emissions in the direction of PSR J1023+0038 with a flux $5 \times 10^{-12} \mathrm{erg} \mathrm{cm}^{-2} \mathrm{~s}^{-1}$. Although the pulsation has not been confirmed in $\gamma$-rays by Tam et al. (2010), its position is in coincidence with the radio position, which lies within the $68 \%$ confidence level error circle of the $\gamma$-ray position. The $\gamma$-ray flux can be estimated as $F_{\gamma} \sim L_{\gamma} / \delta \Omega d^{2} \sim 10^{-9} \mathrm{erg} \mathrm{cm}^{-2} \mathrm{~s}$, where $L_{\gamma}=6 \times 10^{34} \mathrm{erg} \mathrm{s}^{-1}$, the typical solid angle $\delta \Omega=2$, and the distance $d=1.3 \mathrm{kpc}$ are assumed. The theoretical prediction is found to exceed the measured value, however, the $\gamma$-ray flux is dependent on the viewing geometry. For example, in the outer gap model the $\gamma$-ray emission decreases with decreasing viewing angle as measured from the rotation axis.

Given the constraints on the inclination of the orbital plane of PSR J1023+0038, 34 ${ }^{\circ}$ to $\sim 53^{\circ}$, it is likely that the line of sight will pass through the edge of the $\gamma$-ray beam from the outer gap accelerating region and that the observed $\gamma$-ray flux will be much smaller than $F_{\gamma} \sim 10^{-9} \mathrm{erg} \mathrm{cm}^{-2} \mathrm{~s}^{-1}$. Because the $\gamma$-rays associated with the edge of the beam will be emitted with an accelerating electric field smaller than the typical value described by Equation (1), the energy of the $\gamma$ rays will be smaller than that given by Equation (3). Hence, the recent measurement of $\gamma$-ray radiation in the direction of PSR J1023+0038 can be reconciled with the proposed emission model provided that the line of sight passes through the edge of the $\gamma$-ray beam. With the typical outer gap geometry, the $\gamma$-ray pulse profile is expected to be either a single peak or a double peak with a small phase separation for this viewing orientation (cf. Hirotani 2008).

The secondary pairs produced outside the gap region by the pair-creation process will emit non-thermal X-rays via the synchrotron radiation process with a typical energy of $E_{n, X} \sim 2\left(\Gamma / 10^{3}\right)^{2}\left(B \sin \theta / 10^{5}\right) \mathrm{keV}$, where $\Gamma$ is the typical Lorentz factor of the secondary pairs, $B$ is the magnetic field near the light cylinder, and $\theta$ is the pitch angle. The optical depth of the pair-creation process of a $\gamma$-ray can be estimated as $\tau_{X \gamma} \sim n_{s, X} \sigma_{X \gamma} R_{\mathrm{lc}} \sim 10^{-2}$ with $n_{s, X} \sim L_{s, x} /\left(4 \pi R_{\mathrm{lc}}^{2} c E_{s, X}\right)$ corresponding to the X-ray photon density, where we adopted the observed X-ray luminosity $L_{s, X} \sim 10^{31} \mathrm{erg} \mathrm{s}^{-1}$ and $E_{s, X} \sim 30 \mathrm{eV}$ (Homer et al. 2006). In addition, $\sigma_{X \gamma} \sim \sigma_{T} / 3$ is the cross section of the photon-photon pair creation. The luminosity of these non-thermal X-rays is estimated as

$$
L_{n, X} \sim \tau_{X \gamma} L_{\gamma} \sim 6 \times 10^{32} \mathrm{erg} \mathrm{s}^{-1},
$$

which is consistent with the observations $L_{n, X} \sim 5 \times$ $10^{31} \mathrm{erg} \mathrm{s}^{-1}$ (cf. Homer et al. 2006) assuming that we may be observing the edge of the high-energy emission region. The spectral shape below $E_{n, X} \sim 2 \mathrm{keV}$ will be expressed by the photon index $\Gamma_{n, X} \sim 1.5$, which is near the observed value $\Gamma_{n, X} \sim 1.3$ reported by Homer et al. (2006).

To conclude, it has been shown that the irradiation by $\gamma$-rays from the outer gap can facilitate dissolution of an accretion disk within the assumption that the rotation-powered pulsar is active during a sufficiently low-luminosity level in the quiescent state of a soft X-ray transient system and the heating leads to the escape of disk material from the system. The model has been applied to the recently born MSP PSR J1023+0038, and we have estimated that the $\gamma$-ray luminosity is $\sim 6 \times 10^{34} \mathrm{erg} \mathrm{s}^{-1}$, which is consistent with the irradiated luminosity required to explain the heating of the companion star.

Future $\gamma$-ray observations of the pulsed component are needed to confirm that $\mathrm{J} 1023+3008$ is a $\gamma$-ray emitting pulsar and to place constraints on our theoretical model of the $\gamma$ ray emission, its site of production, and its effectiveness in facilitating the removal of the accretion disk in this system.

This work was supported in part by the Theoretical Institute for Advanced Research in Astrophysics (TIARA) operated under the Academia Sinica Institute of Astronomy \& Astrophysics in Taipei, Taiwan and by NSF AST-0703950 to Northwestern University, and KSC is supported by a GRF grant of Hong Kong Government under HKU700908P.

\section{REFERENCES}

Abdo, A. A., et al. 2009, Science, 325, 848

Archibald, A. M., Kaspi, V., Bogdanov, S., Hessels, J. W. T., Stairs, I. H., Ransom, S. M., \& McLaughlin, M. A. 2010, ApJ, 722, 88

Archibald, A. M., et al. 2009, Science, 324, 1411

Arons, J. 2008, in AIP Conf. Proc. 983, 40 Years of Pulsars: Millisecond Pulsars, Magnetars and More, ed. C. Bassa, et al. (Melville, NY: AIP), 200

Bogdanov, S., Grindlay, J. E., \& van den Berg, M. 2005, ApJ, 630, 1029

Bond, H. E., White, R. L., Becker, R. H., \& O'Brien, M. S. 2002, PASP, 114 1359

Burderi, L., Di Salvo, T., D’Antona, F., Robba, N. R., \& Testa, V. 2003, A\&A, 404, L43

Campana, S., Colpi, M., Mereghetti, S., Stella, L., \& Tavani, M. 1998, A\&AR, 8,279

Cheng, K. S., Ho, C., \& Ruderman, M. 1986a, ApJ, 300, 500

Cheng, K. S., Ho, C., \& Ruderman, M. 1986b, ApJ, 300, 522 
D’Avanzo, P., Campana, S., Casares, J., Covino, S., Israel, G. L., \& Stella, L. 2009, A\&A, 508, 297

Deloye, C. J., Heinke, C. O., Taam, R. E., \& Jonker, P. G. 2008, MNRAS, 391, 1619

Frank, J., King, A., \& Raine, D. 2002, Accretion Power in Astrophysics, (Cambridge: Cambridge Univ. Press)

Grindlay, J. E., Camilo, F., Heinke, C. O., Edmonds, P. D., Cohn, H., \& Lugger, P. 2002, ApJ, 581, 470

Heinke, C. O., Jonker, P. G., Wijnands, R., Deloye, C. J., \& Taam, R. E. 2009, ApJ, 691, 1035

Hirotani, K. 2008, Open Astron. J., arXiv:0809.1283

Homer, L., Szkody, P., Cheng, B., Henden, A., Schmidt, G., Anderson, S. F., Silvestri, N. M., \& Brinkmann, J. 2006, AJ, 131, 562
Jeffrey, L. C. 1986, Nature, 319, 384

Jonker, P. G., Torres, M. A. P., \& Steeghs, D. 2008, ApJ, 680, 615

Kirk, J. G., \& Skjæraasen, O. 2003, ApJ, 591, 366

Lang, K. R. 1999, Astrophysical Formulae (New York: Springer)

Romanova, M. M., Ustyugova, G. V., Koldoba, A. V., \& Lovelace, R. V. E. 2009, MNRAS, 399, 1802

Tam, P.-H., et al. 2010, ApJL, submitted

Thorstensen, J. R., \& Armstrong, E. 2005, AJ, 130, 759

Wang, Z., Archibald, A. M., Thorstensen, J. R., Kaspi, V. M., Lorimer, D. R., Stairs, I., \& Ransom, S. M. 2009, ApJ, 703, 2017

Woudt, P. A., Warner, B., \& Pretorius, M. L. 2004, MNRAS, 351, 1015

Zhang, L., \& Cheng, K. S. 1997, ApJ, 480, 370 